\title{
Eosinophilic fasciitis after parasite infection
}

\author{
Marta Oliveira, Fabia Patinha, Antonio Marinho
}

Centro Hospitalar Baixo Vouga, Aveiro, Portugal

\begin{abstract}
Eosinophilic fasciitis is a systemic inflammatory disease characterized by symmetrical swelling and skin induration of the distal portions of the arms and/or legs, evolving into a scleroderma-like appearance, accompanied by peripheral blood eosinophilia. It is a rare disease with a poorly understood etiology. Corticosteroid treatment remains the standard therapy, either taken alone or in association with an immunosuppressive drug.

This paper presents a case of a male patient with palpebral edema and marked eosinophilia, diagnosed with intestinal parasitic infection in October 2006. He was treated with an antiparasitic drug, but both the swelling and the analytical changes remained. This was followed by a skin and muscle biopsy, which turned out to be compatible with eosinophilic fasciitis. There was progressive worsening of the clinical state, with stiffness of the abdominal wall and elevated inflammatory parameters, and the patient was referred to the Immunology Department, medicated with corticosteroids and methotrexate. Over the years there were therapeutic adjustments and other causes were excluded. Currently the patient continues to be monitored, and there is no evidence of active disease.

The case described in this article is interesting because of the diagnosis of eosinophilic fasciitis probably associated/coexisting with a parasite infection. This case report differs from others in that there is an uncommon cause associated with the onset of the disease, instead of the common causes such as trauma, medication, non-parasitic infections or cancer.
\end{abstract}

Key words: eosinophilic fasciitis, antirheumatic agents, rheumatology, allergy, immunology.

\section{Introduction}

Eosinophilic fasciitis (EF) is a rare disease with a poorly understood etiology. The syndrome of diffuse fasciitis and eosinophilia was first described in 1974 by Shulman, characterized by a symmetrical and painful swelling with a progressive induration of the skin and soft tissues, also known as Shulman's syndrome [1, 2]. Since then, over 300 cases have been reported.

In the absence of international diagnostic criteria, diagnosis of EF is based on a full-thickness skin/fascia/muscle biopsy, with an inflammatory infiltration associated with characteristic abnormalities and a thickened fascia. Peripheral eosinophilia is present in $63-93 \%$ of patients, although it is not mandatory for EF diagnosis [3].

Therapeutic management of EF is commonly based on steroids having a partial or complete response in just over $60 \%$ of patients [3]. The use of immunosuppressive drugs (methotrexate, ciclosporin and, more recently, rituximab) is not codified, but is justified mostly due to failure or dependence on high doses of steroids [3-5].

We present a case of EF after parasite infection, an unusual diagnosis, which explains the skin changes and peripheral blood eosinophilia in this patient. It is important to raise clinical awareness of this rare disease; therefore we describe typical findings at physical examination, distinguishing it from clinically similar diseases, such as systemic scleroderma.

\section{Case report}

The study focuses on a previously healthy 52-yearold man who went to the hospital in October 2006 due to a localized pain in the right hypochondrium radiating

Address for correspondence:

Marta Oliveira, Centro Hospitalar Baixo Vouga Av. Artur Ravara, 3814-501, Aveiro, Portugal, e-mail: martasoliv@gmail.com

Submitted: 14.01.2016; Accepted: 25.02.2016 
to the ipsilateral shoulder. The analytic results showed hypereosinophilia (4100/ $\mu$ l, $46 \%$ of the lymphocytes), with normal chest $X$-ray and electrocardiogram. Bilateral edema of the lower limbs and eyelids lasting five days was also reported, but there were no respiratory or urinary complaints.

He had stable vital signs with normal cardiopulmonary auscultation, with a soft, depressible and painless abdomen, as well as slight bilateral eyelid edema and bilateral ankle edema.

He was hospitalized in order to clarify the diagnosis of eosinophilia and edema. There were no alterations in leukocyte count apart from isolated eosinophilia. C-reactive protein was $2.9 \mathrm{mg} / \mathrm{dl}$ and the sedimentation rate was $15 \mathrm{~mm} / \mathrm{h}$. The peripheral blood smear, electrophoresis of proteins and proteinuria tests showed values within the parameters. The immunology study, thyroid function and respective antibodies, antinuclear antibodies (ANAs) and antineutrophil cytoplasmic antibodies (ANCA) were negative. Transthoracic echocardiography was performed, as well as abdominal ultrasound with liver and kidney examination, which proved to be normal. The stool test revealed the presence of many Ascaris lumbricoides eggs. The patient was discharged with the diagnosis of parasite infection, treated with $75 \mathrm{mg}$ of hydroxyzine daily for one week and $200 \mathrm{mg}$ of mebendazole for 3 days.

Three weeks later, in November 2006, the patient was hospitalized again due to the persistence of edema. By that time it was generalized, without palpable lymphadenopathy or organomegaly, although the remaining physical examination was normal. The stool test showed some helminth eggs. The analytical study of tumors and viral markers showed no changes except for the persistence of moderate eosinophilia. He underwent thoracoabdominal computed tomography (CT) and repeated the echocardiography, but these tests did not reveal any significant changes. The gastroscopy and colonoscopy (with duodenal biopsy) showed no abnormalities. A liver biopsy was performed, which showed signs of consistent nonspecific reactive hepatitis. He was discharged with a diagnosis of liver fascioliasis, treated with furosemide and praziquantel, and underwent a skin and muscle biopsy one week later.

The patient was hospitalized for a third time in early December due to an infection in the muscle biopsy scar in his leg, as well as persistent edema, in spite of diuretic treatment, and eosinophilia. This time the analysis of the stools was negative for parasite eggs. Histology of skin and muscle showed signs consistent with eosinophilic fasciitis: normal epidermis, sclerotic dermis and fibrotic septae with inflammatory infiltrate composed of lymphocytes, eosinophils, plasma cells, and histiocytes, and thick fascia with sclerosis. He was treated with albendazole and prednisolone $150 \mathrm{mg}$ daily for 3 days, with a good response after being re-evaluated, and the prescription was reduced to $60 \mathrm{mg}$ of prednisolone per day.

Analysis showed persistently high inflammatory markers and rigidity of the abdominal wall, with functional impairment; therefore the patient was sent to the Immunology Unit in 2007, with typical EF features: involvement of the deep fascia of the forearms and arms, torso and abdomen, apparently still in the inflammatory phase. He showed signs of chronicity in the upper limbs, with venous furrowing but no "preacher's hand", a sign of good therapeutic response. He began immunosuppressive therapy with methotrexate $15 \mathrm{mg}$ weekly, with posterior reduction of corticosteroid to $15 \mathrm{mg}$ per day as well as prophylaxis of osteoporosis. Two months later the cutaneous inflammatory signs (orange peel) had decreased significantly, although he retained seemingly irreversible thickening in the upper limbs and abdominal wall. Having moderately elevated inflammatory parameters, he was treated with methotrexate $17.5 \mathrm{mg}$ weekly, followed by reduction of the prednisolone to $5 \mathrm{mg}$ per day.

The patient continued vigilance in the Immunology Department. Due to the persistence of inflammatory cutaneous signs in the trunk, as well as functional limitation in 2012, the dose of methotrexate was increased to $30 \mathrm{mg}$ daily, associated with $20 \mathrm{mg}$ per day of prednisolone and azathioprine. The patient showed progressive improvement in 2013 with no functional limitations. He was prescribed methotrexate $15 \mathrm{mg}$ weekly, azathioprine $(50 \mathrm{mg} /$ day) and prednisolone $10 \mathrm{mg}$. In 2014 the patient continued a good evolution without any complications, with negative inflammatory markers and without eosinophilia.

In 2015 the patient showed minor sequelae of abdominal and lumbar paraspinal fasciitis, without functional limitation or orange peel aspect (Figs. 1 and 2). In order to eliminate a paraneoplastic syndrome, a thoracic computerized axial tomography scan was performed, which revealed no changes. Although the abdominal ultrasonography showed thickening of the gastric wall, the endoscopy examination was normal, and the analysis showed no evidence of inflammatory activity or eosinophilia.

\section{Discussion}

Eosinophilic fasciitis is a systemic inflammatory disease characterized by symmetrical swelling and skin induration of the distal portions of the arms and/or legs, with hyperpigmentation of the extremities, and it is accompanied by peripheral blood eosinophilia, and hence is compatible with the case of this patient $[6,7]$. The dis- 


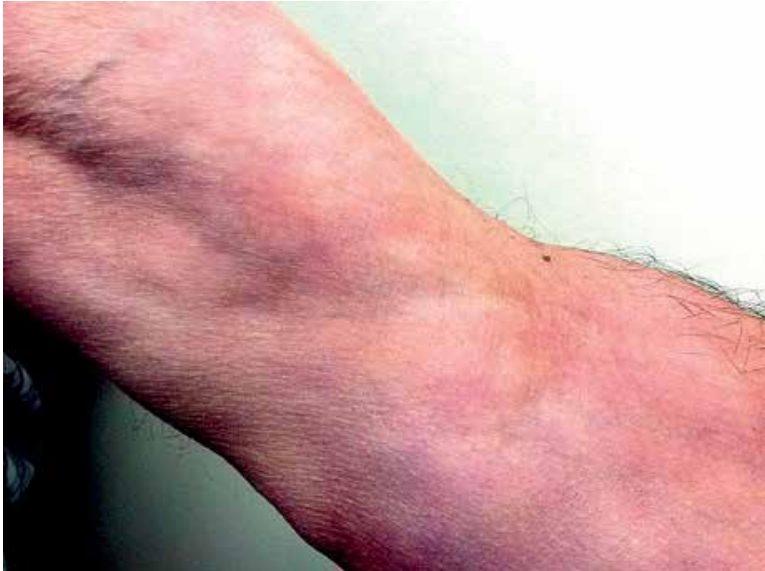

Fig. 1. The "groove sign" on the right forearm of the patient.

ease appears in 40- to 50-year-old men and women with no sex bias, but it is seen earlier in men [8]. The pathophysiology is unknown and unclear. Shakoory et al. [9] suggest a role for mast cells and cytokines. Abnormal circulating T-cell clones and increased interleukin-5 production could be responsible for the eosinophilia and eosinophil-mediated tissue injury, as suggested by French et al. [10]. First there is edema of the affected areas, sore to the touch. Eventually, the skin develops a "peau d'orange" aspect ("orange peel skin"), which may become indurated and evolve into a scleroderma-like appearance. Physical examination classically reveals signs of venous furrowing, the "groove sign" (Fig. 1). The induration and sclerosis of subcutaneous tissue can result in contractures in the joints. In EF it is possible to make folds on the skin (Fig. 2), and the hands and feet usually show no signs of sclerodactyly, as opposed to systemic sclerosis. In addiction to these features, the presence of the Raynaud phenomenon and the involvement of internal organs are uncommon. Inflammatory arthritis can be seen as an extracutaneous manifestation [6].

Laboratory analysis in EF typically shows peripheral blood eosinophilia, an elevated erythrocyte sedimentation rate and hypergammaglobulinemia [2]. However, the data do not always correlate with the disease. The laboratory abnormalities can be transitory and, therefore, are not mandatory for the diagnosis. In this case, the eosinophilia was present from the start, but the initial parasite infection may have delayed the final diagnosis.

A wide variety of infectious agents, almost exclusively helminth (worm) parasites, elicit eosinophilia [11]; only relatively few, however, elicit a sustained, marked increase in eosinophil levels [12]. The pattern and degree of eosinophilia in parasitic infections are determined by the development, migration, and distribution of the par-

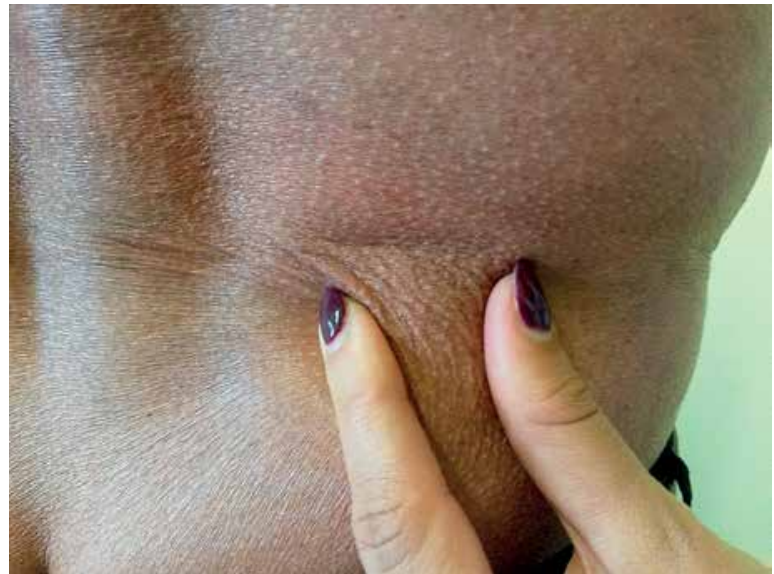

Fig. 2. It is possible to make folds on the patient's skin, which nowadays does not have the "peau d'orange" aspect.

asite within the host as well as by the host's immune response. Parasites tend to elicit marked eosinophilia when they or their products come into contact with immune effector cells in tissues, particularly during migration. Therefore, eosinophilia is highest among parasites with a phase of development that involves migration through tissue (e.g., trichinosis, ascariasis, gnathostomiasis, filarial parasites) [13]. In this patient, the stool test revealed eggs of Ascaris lumbricoides, which leads us to think that the EF in this patient very likely results from the parasitic infection and is not just a coexistence of two unrelated disorders.

Ascaris lumbricoides is one of the most common intestinal nematodes, transmitted primarily through the fecal-oral route. Adult worms in the bowel lumen cause no symptoms or only mild abdominal pain, nausea or diarrhea, which are nonspecific findings common in other helminth infections [14].

A full thickness incisional skin biopsy (down to superficial muscle) is needed to confirm the diagnosis, and in this case the result of the biopsy was consistent with the suspected disease.

Histologically, pronounced thickening of the subcutaneous fascia is generally considered to be the most distinctive feature of eosinophilic fasciitis [6]. The histological abnormalities change while the disease progresses. Early in the course of the disease the deep fascia and lower tissues are edematous and infiltrated with lymphocytes, plasma cells, histiocytes and eosinophils. In a later stage these structures and eventually the dermis become collagenized, thickened and sclerotic. The fascial thickening can be seen with magnetic resonance imaging [15].

Systemic use of glucocorticoids is generally considered the chosen therapeutic agent. Usually, a high-dose 
regimen is used. In a French study with 34 adult patients with biopsy-proven EF, the authors concluded that corticosteroid treatment remains the standard therapy for EF, taken alone or in association with an immunosuppressive drug; methylprednisolone pulses at initiation of treatment are associated with a better outcome. An immunosuppressive drug, usually methotrexate, might be useful as a second-line therapy [4]. There are few controlled trials, and these practical conclusions should be confirmed by prospective and multicentre studies.

There are various therapies which have also been applied, such as D-penicillamine, chloroquine, cimetidine, azathioprine, cyclosporine, photochemotherapy and infliximab [5, 8]. The assessment of long-term treatment effects is difficult to do, since spontaneous remission of the disease may occur.

Complications of EF are contractures in the joints, peripheral polyneuropathy and a compartment syndrome. None of these were found in this patient. Seldom, a malignancy can present itself as EF as a paraneoplastic syndrome, in most cases before the cancer is diagnosed. Some particular associations with hematolymphoid malignancies have been described [7, 16]. There are scarce reports linking EF with solid malignancies. These include prostate cancer, choroidal melanoma, lung cancer, and breast cancer [17]. Relative unresponsiveness to glucocorticoid therapy is reported to be a red flag for cancer-associated eosinophilic fasciitis [7].

\section{Summary}

In conclusion, EF is a rare skin sclerosis and should be considered in the differential diagnosis in the case of unknown swelling and pain. Although laboratory parameters may not correlate with the evolution of the disease, a full-thickness skin/fascia/muscle biopsy and magnetic resonance imaging may significantly contribute to the diagnosis. This case illustrates the importance of maintaining surveillance of these patients for a medication adjustment, in order to control the inflammatory signs and cutaneous manifestations. It is also important to rule out paraneoplastic syndrome. In general, patients benefit from a high-dose steroid therapy.

The authors declare no conflict of interest.

\section{References}

1. Shulman LE. Diffuse fasciitis with hypergammaglobulinemia and eosinophilia: a new syndrome? J Rheumatol 1974; 11: 569-570.

2. Shulman LE. Diffuse fasciitis with eosinophilia: a new syndrome? Trans Assoc Am Physicians 1975; 88: 70-86.
3. Endo Y, Tamura A, Matsushima Y, et al. Eosinophilic fasciitis: report of two cases and a systematic review of the literature dealing with clinical variables that predict outcome. Clin Rheumatol 2007; 26: 1445-1451.

4. Lebeaux D, Francès C, Barete S. Eosinophilic fasciitis (Shulman disease): new insights into the therapeutic management from a series of 34 patients. Rheumatology 2012; 51: 557-561.

5. Khanna D, Agrawal H, Clements PJ. Infliximab may be effective in the treatment of steroid-resistant eosinophilic fasciitis: report of three cases. Rheumatology 2010; 49: 1184-1188.

6. Tsoi KL, Custers M, Bij de Vaate L, Jacobs JW. Eosinophilic fasciitis. BMJ Case Reports 2012; DOI: 10.1136/bcr.2012.6158.

7. Haddad H, Sundaram S, Magro C, Gergis U. Eosinophilic fasciitis as a paraneoplastic syndrome, a case report and review of the literature. Hematol Oncol Stem Cell Ther 2014; 7: 90-92.

8. Antic M, Lautenschlager S, Itin PH. Eosinophilic fasciitis 30 years after - what do we really know? Report of 11 patients and review of the literature. Dermatology 2006; 213: 93-101.

9. Shakoory B, Fitzgerald SM, Lee SA, et al. The role of human mast cell-derived cytokines in eosinophil biology. J Interferon Cytokine Res 2004; 24: 271-281.

10. French LE, Shapiro M, Junkins-Hopkins JM, et al. Eosinophilic fasciitis and eosinophilic cellulitis in a patient with abnormal circulating clonal T cells: increased production of interleukin 5 and inhibition by interferon alfa. J Am Acad Dermatol 2003; 49: 1170-1174.

11. Moore TA, Nutman TB. Eosinophilia in the returning traveler. Infect Dis Clin North Am 1998; 12: 503-521.

12. Wilson ME. A World Guide to Infections: Diseases, Distributions, Diagnosis. Oxford University Press, New York 1991; 164.

13. Weller PF. Eosinophilia in travelers. Med Clin North Am 1992; 76: 1413-1432.

14. Haque R. Human Intestinal Parasites. J Health Popul Nutr 2007; 25: 387-391.

15. De Clerck LS, Degryse HR, Wouters E, et al. Magnetic resonance imaging in the evaluation of patients with eosinophilic fasciitis. J Rheumatol 1989; 16: 1270-1273.

16. Naschitz JE, Rosner I, Rozenbaum M, et al. Rheumatic syndromes: clues to occult neoplasia. Semin Arthritis Rheum 1999; 29: 43-55.

17. Veyssier-Belot C, Zuech P, Lumbroso-Le Rouic L, et al. Eosinophilic fasciitis and metastatic choroidal melanoma: a paraneoplastic syndrome? Rev Med Interne 2008; 29: 1013-1016. 